TDI and clock noise removal for the split interferometry configuration of LISA

This article has been downloaded from IOPscience. Please scroll down to see the full text article.

2012 Class. Quantum Grav. 29205003

(http://iopscience.iop.org/0264-9381/29/20/205003)

View the table of contents for this issue, or go to the journal homepage for more

Download details:

IP Address: 194.94.224.254

The article was downloaded on 03/09/2012 at 11:25

Please note that terms and conditions apply. 


\title{
TDI and clock noise removal for the split interferometry configuration of LISA
}

\author{
Markus Otto $^{1,2}$, Gerhard Heinzel ${ }^{1,2}$ and Karsten Danzmann ${ }^{1,2}$ \\ ${ }^{1}$ Max Planck Institute for Gravitational Physics (Albert Einstein Institute) and Institute for \\ Gravitational Physics, Leibniz University of Hannover, Callinstraße 38, 30167 Hannover, \\ Germany \\ ${ }^{2}$ QUEST Centre for Quantum Engineering and Space-Time Research, Leibniz University of \\ Hannover, Germany \\ E-mail: markus.otto@aei.mpg.de
}

Received 24 January 2012, in final form 9 July 2012

Published 23 August 2012

Online at stacks.iop.org/CQG/29/205003

\begin{abstract}
Laser phase noise is the dominant noise source in the on-board measurements of the space-based gravitational wave detector LISA (Laser Interferometer Space Antenna). A well-known data analysis technique, the so-called timedelay interferometry (TDI), provides synthesized data streams free of laser phase noise. At the same time, TDI also removes the next largest noise source: phase fluctuations of the on-board clocks which distort the sampling process. TDI needs precise information about the spacecraft separations, sampling times and differential clock noise between the three spacecrafts. These are measured using auxiliary modulations on the laser light. Hence, there is a need for algorithms that account for clock noise removal schemes combined with TDI while preserving the gravitational wave signal. In this paper, we will present the mathematical formulation of the LISA-like data streams and discuss a compliant algorithm that corrects for both clock and laser noise in the case of a rotating, non-breathing LISA constellation. In contrast to previous papers, we consider the current optical bench design (split interferometry configuration), i.e. the test mass readout is done by the local oscillators only, instead of reflecting the weak inter-spacecraft light off the test mass. Furthermore, the absolute order of laser frequencies is taken into account and it can be shown that the TDI equations remain invariant. This is a crucial issue and was, up to now, completely neglected in the analysis.
\end{abstract}

PACS numbers: 04.80.Nn, 07.60.Ly, 07.87.+v, 42.60.Fc, 42.60.Mi, 95.55.Br, 95.55.Sh, 95.55.Ym, 95.75.Kk

(Some figures may appear in colour only in the online journal) 


\section{Introduction}

The Laser Interferometer Space Antenna (LISA) is an international scientific space mission $[1,2]$ to be launched within the next decade. This paper uses the 2010 LISA design. The goal of LISA is to measure gravitational waves in the $\mathrm{mHz}$ frequency band, produced, for example, by white dwarf binary systems or by coalescing supermassive black hole binaries.

The LISA consists of three spacecraft (S/C) forming a nearly equilateral triangle with 5 million kilometre armlength. Each $\mathrm{S} / \mathrm{C}$ carries two free-falling test masses (TM) marking the endpoints of the arms, and two identical optical benches (OB) connected via optical fibres to one laser source per $\mathrm{OB}$ and to each other. The laser beam is sent through a $40 \mathrm{~cm}$ telescope to the distant spacecraft where heterodyne interferometry between the incoming and the local laser beam is performed. This measurement produces a beatnote, the phase of which carries information about the gravitational waves that modulate the light travel time between the satellites. However, many noise sources also enter the measured data. The dominant noise source is the laser phase noise, which enters via the armlength difference (up to $1 \% \approx 50000 \mathrm{~km}$ ) and completely swamps the gravitational wave signal, even after various laser stabilization schemes have been applied [3]. To remove the laser phase noise, a post-processing method called time-delay interferometry (TDI), first proposed by [4], has to be applied. TDI synthesizes virtual equal armlength interferometers by combining the measured data of the spacecraft after time-shifting by multiples of the light travel times in the arms. Therefore, the exact absolute armlengths need to be determined by a separate ranging method (approximately metre accuracy) between the spacecrafts $[5,6]$.

Before all further processing, the beatnote must be digitized via an analogue-digital converter (ADC). This ADC is triggered by a stable clock, a so-called ultrastable oscillator (USO). Each spacecraft carries a separate free-running USO. This USO itself is limited by noise, which introduces clock jitter in the synthesized data streams and furthermore in the ranging signal, thereby affecting the arm length measurements. However, the data streams of the carrier-carrier beatnote phase alone cannot provide enough information to cancel the clock noise of the USO. Hence, a clock tone transfer chain was proposed in [7] using sideband (SB) modulations with amplified clock noise on the outgoing light. After defining one of the clocks as a reference, these SB modulations yield sufficient data to completely remove the clock noise and allow correction of relative clock drifts with respect to the master clock.

Some changes in the OB design (that is, the incoming light from the distant $\mathrm{S} / \mathrm{C}$ is not reflected off the TM as opposed to the previous design) make it necessary to reconsider in detail the LISA-like data streams and TDI equations $[13,14]$. We will discuss the OB design and its consequences for the LISA-like data streams in the case of a rotating, non-flexing LISA constellation. This paper presents the main challenge to find an algorithm that removes the main noise sources while preserving the gravitational wave signal. Additionally, there is another issue: up to now, the introduction of an absolute ordering of the on-board laser frequencies was neglected in TDI papers which we redress here and show that the standard TDI equations will not change.

This paper is organized as follows. In section 2, the notation of TDI is introduced and the main LISA-like data streams on the $\mathrm{S} / \mathrm{C}$ for the split interferometry configuration layout will be presented. Section 3 shows how to remove the main noise sources in the LISA data streams analytically, followed by a discussion and outlook in section 4. 


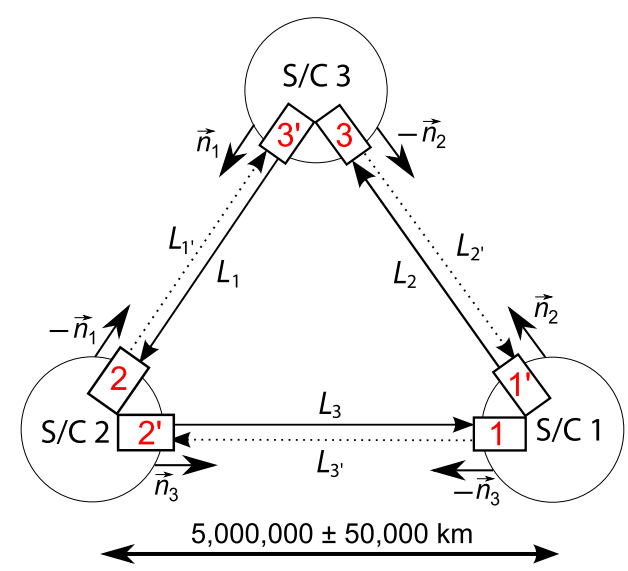

Figure 1. Notation of LISA lasers and links. Note that $\mathbf{n}_{i^{\prime}}=-\mathbf{n}_{i}$.

\section{LISA-like data streams}

\subsection{Notation}

In the common TDI papers [4, 8-10], various notations coexist. We will introduce the following notation according to figure 1 and refer to it in the following.

Each of the three spacecrafts carries two nearly identical OB, where one is denoted with unprimed numbers $(1,2,3)$ and the other by primed numbers $\left(1^{\prime}, 2^{\prime}, 3^{\prime}\right)$, as shown in figure 1 . The 'armlengths' of the triangle in terms of the light travel time $t_{i}, L_{i}=c t_{i}$, are marked as follows: the opposed side of $\mathrm{S} / \mathrm{C} i$ is denoted by $L_{i}$ and $L_{i^{\prime}}$, respectively, depending on the direction of light travel (counter-clockwise or clockwise). We thus introduce two kinds of indices: indices without a prime (e.g. $i$ ) count $1,2,3$ and indices with a prime (e.g. $\left.i^{\prime}\right)$ count $1^{\prime}, 2^{\prime}, 3^{\prime}$. We also introduce the unit direction vectors $\mathbf{n}$ pointing along the LISA arms. $\mathbf{n}_{i}$ points along the arm $i$ in the counter-clockwise direction, while $\mathbf{n}_{i^{\prime}}=-\mathbf{n}_{i}$ points in the opposite direction.

Furthermore, it is convenient to define the time-delay operators $\mathcal{D}$ which act on the continuous functions $f(t)$ as [11]

$$
\mathcal{D}_{k} f(t)=f\left(t-\frac{L_{k}}{c}\right), \quad \mathcal{D}_{j} \mathcal{D}_{k} f(t)=f\left(t-\frac{L_{j}}{c}-\frac{L_{k}}{c}\right),
$$

where $j$ and $k$ can take the values of $1,1^{\prime}, 2,2^{\prime}, 3,3^{\prime}$. Powers of time-delay operators mean multiple applications of $\mathcal{D}$, e.g. $\mathcal{D}_{j}^{2} f(t)=\mathcal{D}_{j} \mathcal{D}_{j} f(t)=f\left(t-2 L_{j} / c\right)$. These definitions are sufficient in the case of a non-flexing, rotating LISA constellation. In the case of arm flexing, the armlengths $L_{k}$ are time dependent and the time-delay operators need to be defined in a more complex way. We consider only time-independent armlengths in this paper. However, due to the Sagnac effect, the rotation of the rigid satellite constellation makes it necessary to distinguish between $L_{i}$ and $L_{i^{\prime}}$; hence $\mathcal{D}_{i} f(t) \neq \mathcal{D}_{i^{\prime}} f(t)$.

In general, one has to consider carefully the direction definitions. We introduce a consistent description according to figure 2 and consider for the moment only one LISA arm and the optical phase shift experienced by the light travelling along the arm due to the OB displacement noise $\boldsymbol{\Delta}$ relative to an inertial frame (if the bench moves, the TM will still follow the geodesics). Therefore, the scalar product takes care of the correct directions. The change in separation measured as an optical phase shift from OB $2^{\prime}$ to 1 is then given by 


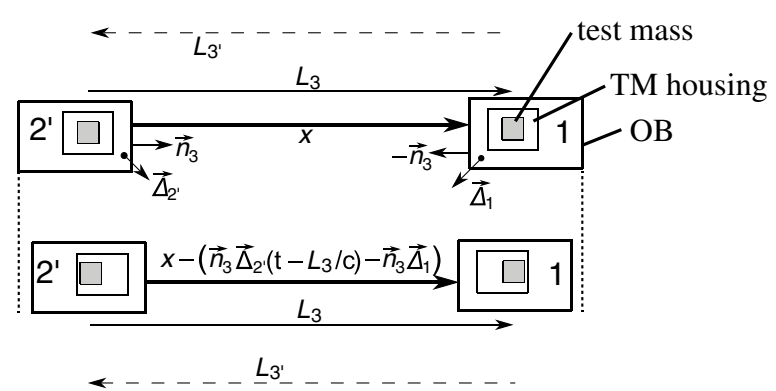

Figure 2. Direction definition for one LISA arm at two different times. $L_{3}$ and $L_{3^{\prime}}$ are the distances in terms of the light travel time between the TM (grey boxes) dependent on the direction; $x<L_{3}$ denotes the distance between the OB $2^{\prime}$ and 1 (outer boxes).

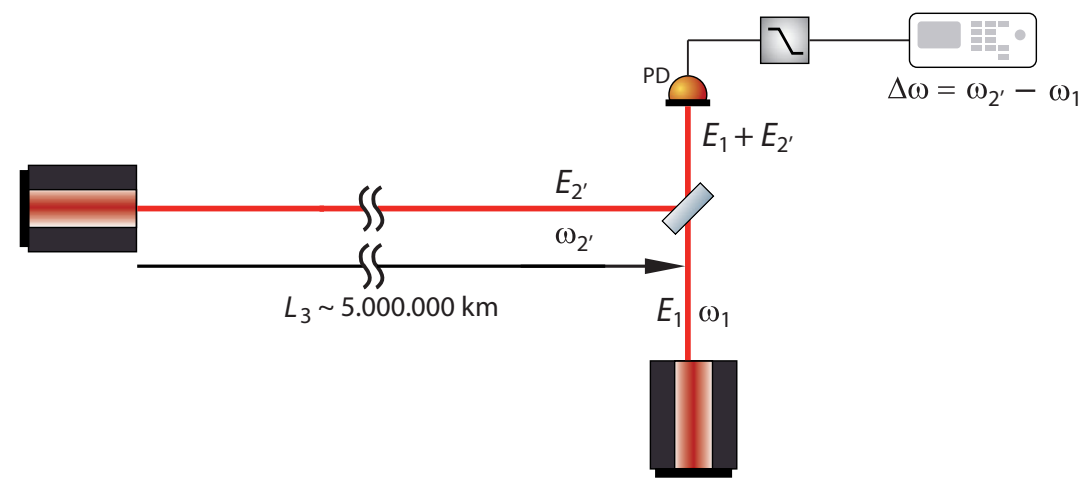

Figure 3. One schematic LISA arm. $E_{2^{\prime}}$ is the electric field of the incoming light, $E_{1}$ of the local laser. The photodetector (PD) measures the sum of the power of the electric fields, i.e. $P \sim\left|E_{1}+E_{2^{\prime}}\right|^{2}$.

$\frac{2 \pi}{\lambda_{2^{\prime}}} \mathbf{n}_{3} \cdot \mathcal{D}_{3} \boldsymbol{\Delta}_{2^{\prime}}(t)-\frac{2 \pi}{\lambda_{2^{\prime}}} \mathbf{n}_{3} \cdot \boldsymbol{\Delta}_{1}(t)$, as illustrated in figure 2. $\lambda_{2^{\prime}}$ denotes the wavelength of the sensing laser light.

\subsection{Phasemeter signals}

One has to define carefully the phasemeter signals and their phase signs. Therefore, we will consider one LISA arm as shown in figure 3 and compute the measured electric field at one photodetector.

Neglecting geometric and amplitude factors, the electric field from the local laser onboard OB 1 can be written as

$$
E_{1}=\exp \left(\mathrm{i}\left[\omega_{1} t-k_{1} u+\psi_{1}\right]\right),
$$

where $\psi_{1}$ is an additional phase containing laser phase noise and optical phase shift introduced by pathlength variation, e.g., due to the temperature change. Here, $u$ denotes the optical pathlength from the local laser to the beamsplitter. Similarly, the incoming electric field can be written as

$$
E_{2^{\prime}}=\exp \left(\mathrm{i}\left[\omega_{2^{\prime}} t-\mathbf{k}_{2^{\prime}} \cdot \mathbf{n}_{3} L_{3}+\psi_{2^{\prime}}\right]\right) .
$$




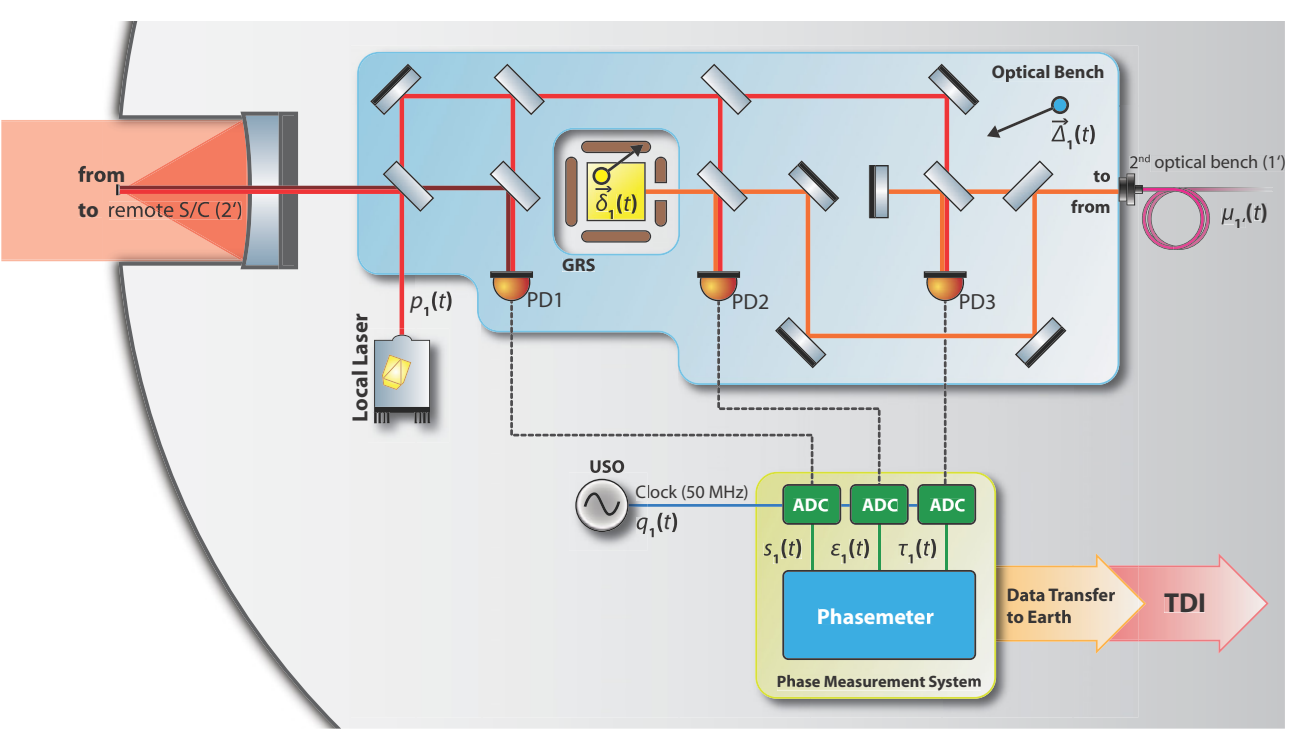

Figure 4. LISA OB setup, shown for bench ' 1 ' as an example. Telescope (left), laser, phasemeter and $\mathrm{OB}$ are all fixed w.r.t. each other. In contrast to the previous TDI literature, here we use the OB layout of the year 2010 (baseline since 2006), the so-called split interferometry configuration.

Note that the frequency $\omega_{2^{\prime}}$ of $E_{2^{\prime}}$ is slightly different to $\omega_{1}$ of $E_{1}$ (by an amount of $\Delta \omega:=\omega_{2}-\omega_{1}$, assumed positive here) due to offset frequency locking and Doppler shifts. $\psi_{2^{\prime}}$ contains again laser phase noise and optical phase shift by pathlength noise from OB $2^{\prime}$, both time-delayed by $L_{3} / c$, and the phase shift due to gravitational waves.

The photodiode measures the power of the sum of incoming and local laser light fields, i.e. $P \sim|E|^{2}=\left|E_{2^{\prime}}+E_{1}\right|^{2}$. Hence, after low-pass filtering, the heterodyne signal is

$$
\begin{aligned}
P & \sim \cos \left(\left[\omega_{2^{\prime}} t-\mathbf{k}_{2^{\prime}} \cdot \mathbf{n}_{3} L_{3}+\psi_{2^{\prime}}\right]-\left[\omega_{1} t-k_{1} u+\psi_{1}\right]\right) \\
& =: \cos (\Delta \omega t+\varphi) .
\end{aligned}
$$

This equation gives the change in phase due to a pathlength change and is commonly referred to as the beatnote. The phase $\varphi$ is the result of the processing of the beatnote in the phasemeter, and its sign is defined by this equation. $\varphi$ itself is given by

$$
\varphi=\psi_{2^{\prime}}-\psi_{1}+k_{1} u-\mathbf{k}_{2^{\prime}} \cdot \mathbf{n}_{3} L_{3}
$$

and will be further specified in the following section. $\varphi$ reverses its sign if $\Delta \omega<0$. Similar calculations can be performed for all LISA arms and onboard interferometers.

\subsection{Split interferometry configuration}

Figure 4 shows a sketch of one LISA OB. The incoming laser beam from the distant S/C (from the left) is interfered with a local oscillator (LO) from the OB. The TM motion is read out by a separate interferometer using laser light from the adjacent $\mathrm{OB}$ on the common $\mathrm{S} / \mathrm{C}$ transmitted through a 'backlink' fibre in addition to the same LO.

Three measurements are performed per OB. First, the incoming laser beam from the distant S/C is interfered with a LO (left photodiode PD1 in figure 4). Due to the fact that the incoming laser light carries information about a passing gravitational wave, this measurement is referred to as the 'science' interferometer signal, $s_{1}(t)$. Next, the TM motion is read out 
interferometrically. For this purpose, the LO is interfered with the LO from the adjacent OB $1^{\prime}$, delivered through a 'backlink' fibre and reflected off the TM. This interferometric readout $\varepsilon_{1}(t)$ at PD2 is called the TM readout. In the third so-called reference interferometer, both lasers of the adjacent OBs 1 and $1^{\prime}$ are interfered at PD3 and give the reference interferometer output $\tau_{1}(t)$.

We will now describe these signals neglecting any change in the refractive index (e.g. by windows of the TM vacuum chamber), $n \equiv 1$. The science interferometer signal $s_{1}(t)$ expressed as the phase of the beatnote (corresponding to $\varphi$ of equation (3)) contains the laser phase noise (called $\left.p_{i}(t)\right)$ from the distant and local OB $\left(p_{2^{\prime}}\left(t-L_{3} / c\right)=: \mathcal{D}_{3} p_{2^{\prime}}(t)\right.$ and $p_{1}(t)$, respectively). Next, the gravitational phase shift $h_{1}(t)$ collected by the transmitted laser light along $L_{3}$ will enter the data, resulting in

$$
\bar{s}_{1}(t)=\mathcal{D}_{3} p_{2^{\prime}}(t)-p_{1}(t)+h_{1}(t)+n_{1}(t) .
$$

The bar shows that the frequency order of the lasers (one crucial issue discussed in section 2.4) is not considered here. In equation (4), the noise $n_{1}(t)$ still needs to be specified. It contains phase fluctuations due to shot noise and electronic noise of the PD (denoted by $N_{1}^{\mathrm{s}}(t)$ ), optical pathlength noise, $N_{1}^{\mathrm{opt}}(t)$, and OB motion of the local and distant OB measured using the incoming light from laser $2^{\prime}$, resulting in

$\bar{s}_{1}(t)=\mathcal{D}_{3} p_{2^{\prime}}(t)-p_{1}(t)+h_{1}(t)+N_{1}^{\mathrm{opt}}(t)-\frac{2 \pi}{\lambda_{2^{\prime}}} \mathbf{n}_{3} \cdot \mathcal{D}_{3} \boldsymbol{\Delta}_{2^{\prime}}(t)+\frac{2 \pi}{\lambda_{2^{\prime}}} \mathbf{n}_{3} \cdot \boldsymbol{\Delta}_{1}(t)+N_{1}^{\mathrm{s}}(t)$.

This equation holds for an idealized perfect phase measurement at the photodiode. However, for further processing, the data need to be digitized in an ADC triggered from the USO (figure 4), which is itself noisy [12]. Thus, the sampling process introduces the clock noise $q(t)$ to the data and we obtain

$$
\begin{aligned}
\bar{s}_{1}^{\mathrm{c}}(t)=\mathcal{D}_{3} p_{2^{\prime}}(t) & -p_{1}(t)+h_{1}(t)+\alpha_{1} q_{1}(t)+N_{1}^{\mathrm{opt}}(t) \\
& -\frac{2 \pi}{\lambda_{2^{\prime}}} \mathbf{n}_{3} \cdot \mathcal{D}_{3} \boldsymbol{\Delta}_{2^{\prime}}(t)+\frac{2 \pi}{\lambda_{2^{\prime}}} \mathbf{n}_{3} \cdot \boldsymbol{\Delta}_{1}(t)+N_{1}^{\mathrm{s}}(t),
\end{aligned}
$$

where $\alpha_{1}=\frac{\Delta f_{s_{1}}}{f_{\mathrm{ADC}}}$ is the conversion factor from the phase noise $q$ referred to $f_{\mathrm{ADC}}$ to phase noise at the signal frequency, $\Delta f_{s_{1}}=\frac{\left|\omega_{2^{\prime}}-\omega_{1}\right|}{2 \pi}$. The superscript $\mathrm{c}$ in $\bar{s}_{1}^{\mathrm{c}}(t)$ stands for the carrier beatnote (section 2.5).

Next, the TM interferometer signal $\varepsilon_{1}(t)$ contains laser phase noise from OB 1 and $1^{\prime}$ $\left(p_{1}(t)\right.$ and $\left.p_{1^{\prime}}(t)\right)$, shot noise and electronic readout noise $\left(N_{1}^{\varepsilon}(t)\right)$, as well as TM displacement noise, $\boldsymbol{\delta}_{1}(t)$, and OB noise, $\boldsymbol{\Delta}_{1}(t)$ (both relative to $\mathbf{n}_{3} L_{3}$ ), fibre noise, $\mu_{1^{\prime}}(t)$, collected by light coming from $\mathrm{OB} 1^{\prime}$ travelling through the 'backlink' fibre and finally clock noise from the ADC:

$\bar{\varepsilon}_{1}(t)=p_{1^{\prime}}(t)-p_{1}(t)+\beta_{1} q_{1}(t)+\mu_{1^{\prime}}(t)+N_{1}^{\varepsilon}(t)-2\left(\frac{2 \pi}{\lambda_{1^{\prime}}} \mathbf{n}_{3} \cdot \boldsymbol{\delta}_{1}(t)-\frac{2 \pi}{\lambda_{1^{\prime}}} \mathbf{n}_{3} \cdot \boldsymbol{\Delta}_{1}(t)\right)$,

where $\beta_{1}=\frac{\Delta f_{\varepsilon_{1}}}{f_{\mathrm{ADC}}}$ with $\Delta f_{\varepsilon_{1}}=\frac{\left|\omega_{1^{\prime}}-\omega_{1}\right|}{2 \pi}$. The factor of 2 in front of the parentheses in equation (6) comes from the light travelling back and forth to the TM as shown in figure 5.

Finally, the reference IFO output, $\tau_{1}(t)$, contains the beatnote of the lasers of OB 1 and $1^{\prime}$, clock noise, electronic readout noise, $N_{1}^{\tau}(t)$, and fibre noise, $\mu_{1^{\prime}}(t)$, collected by the laser light coming from OB $1^{\prime}$ and is hence given by

$$
\bar{\tau}_{1}(t)=p_{1^{\prime}}(t)-p_{1}(t)+\beta_{1} q_{1}(t)+\mu_{1^{\prime}}(t)+N_{1}^{\tau}(t) .
$$



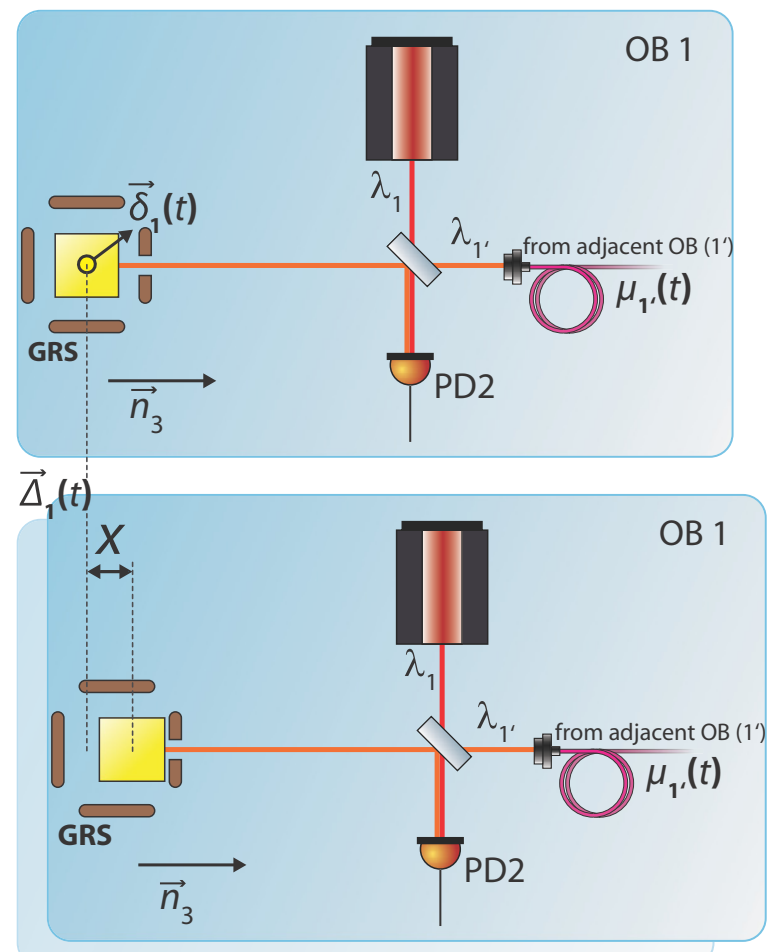

Figure 5. Sketch of the TM interferometer. Light from the adjacent OB $1^{\prime}$, transmitted through a fibre, is used for sensing the TM motion $\delta_{1}(t)$. $x$ denotes the optical pathlength change due to OB motion $\left(+\mathbf{n}_{3} \cdot \boldsymbol{\Delta}_{1}(t)\right)$ and TM motion $\left(-\mathbf{n}_{3} \cdot \boldsymbol{\delta}_{1}(t)\right)$, both entering with a factor of 2 in $\varepsilon_{1}(t)$.

\subsection{Frequency order}

Up to now, we have disregarded one crucial issue that was completely neglected in previous papers: the phasemeter, while reading out the phases of each input signal, interprets all frequencies as positive. Thus, the frequency differences in $\bar{s}^{\mathfrak{c}}(t), \bar{\tau}(t)$ and $\bar{\varepsilon}(t)$ depend on the value of frequencies $v_{i}$. In each data stream, it has to be checked if the frequency difference read out by the phasemeter is positive or negative. In the negative case, the input has to be inverted. We therefore introduce a factor

$$
\theta_{i j}= \begin{cases}+1 & \text { if } v_{i}>v_{j} \\ -1 & \text { if } v_{i}<v_{j}\end{cases}
$$

to keep track of the sign of the measurement, valid for one segment of the frequency plan, typically several days to weeks. We obtain the following system of equations:

$$
\begin{aligned}
& s_{1}^{\mathrm{c}}(t)=\theta_{2^{\prime} 1}\left[\mathcal{D}_{3} p_{2^{\prime}}(t)-p_{1}(t)+h_{1}(t)+N_{1}^{\mathrm{opt}}(t)-\frac{2 \pi}{\lambda_{2^{\prime}}} \mathbf{n}_{3} \cdot \mathcal{D}_{3} \boldsymbol{\Delta}_{2^{\prime}}(t)+\frac{2 \pi}{\lambda_{2^{\prime}}} \mathbf{n}_{3} \cdot \boldsymbol{\Delta}_{1}(t)\right] \\
& +\alpha_{1} q_{1}(t)+N_{1}^{\mathrm{s}}(t) \\
& \tau_{1}(t)=\theta_{1^{\prime} 1}\left[p_{1^{\prime}}(t)-p_{1}(t)+\mu_{1^{\prime}}(t)\right]+\beta_{1} q_{1}(t)+N_{1}^{\tau}(t), \\
& \varepsilon_{1}(t)=\theta_{1^{\prime} 1}\left[p_{1^{\prime}}(t)-p_{1}(t)+\mu_{1^{\prime}}(t)-2\left(\frac{2 \pi}{\lambda_{1^{\prime}}} \mathbf{n}_{3} \cdot \boldsymbol{\delta}_{1}(t)-\frac{2 \pi}{\lambda_{1^{\prime}}} \mathbf{n}_{3} \cdot \boldsymbol{\Delta}_{1}(t)\right)\right] \\
& +\beta_{1} q_{1}(t)+N_{1}^{\varepsilon}(t)
\end{aligned}
$$




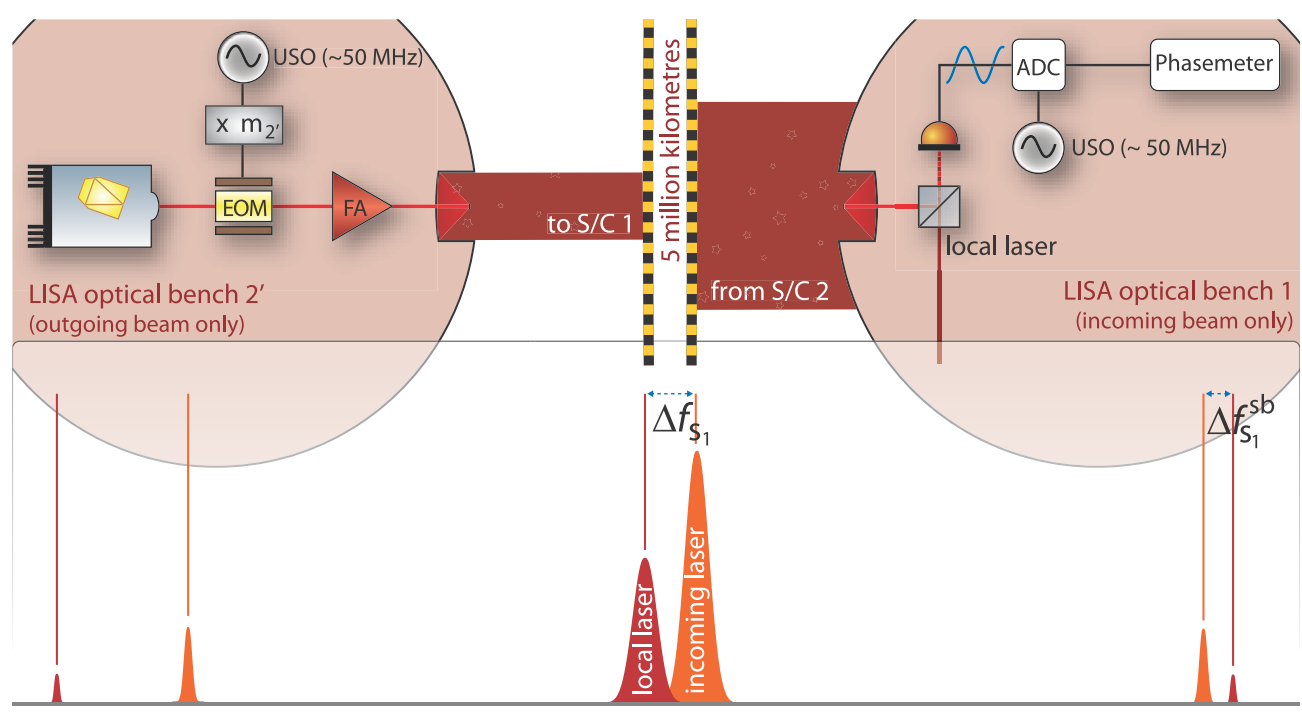

Figure 6. A rough sketch of the clock tone transfer chain. The modulation scheme of the outgoing light from S/C 1 to S/C 2 is similar. Below, the carrier and SB of incoming and local laser light are shown in Fourier space.

$$
\begin{aligned}
& s_{1^{\prime}}^{\mathrm{c}}(t)=\theta_{31^{\prime}}\left[\mathcal{D}_{2^{\prime}} p_{3}(t)-p_{1^{\prime}}(t)+h_{1^{\prime}}(t)+N_{1^{\prime}}^{\mathrm{opt}}(t)+\frac{2 \pi}{\lambda_{3}} \mathbf{n}_{2} \cdot \mathcal{D}_{2^{\prime}} \boldsymbol{\Delta}_{3}(t)-\frac{2 \pi}{\lambda_{3}} \mathbf{n}_{2} \cdot \boldsymbol{\Delta}_{1^{\prime}}(t)\right] \\
& +\alpha_{1^{\prime}} q_{1}(t)+N_{1^{\prime}}^{\mathrm{s}}(t) \\
& \tau_{1^{\prime}}(t)=\theta_{11^{\prime}}\left[p_{1}(t)-p_{1^{\prime}}(t)+\mu_{1}(t)\right]+\beta_{1^{\prime}} q_{1}(t)+N_{1^{\prime}}^{\tau}(t), \\
& \varepsilon_{1^{\prime}}(t)=\theta_{11^{\prime}}\left[p_{1}(t)-p_{1^{\prime}}(t)+\mu_{1}(t)+2\left(\frac{2 \pi}{\lambda_{1}} \mathbf{n}_{2} \cdot \boldsymbol{\delta}_{1^{\prime}}(t)-\frac{2 \pi}{\lambda_{1}} \mathbf{n}_{2} \cdot \boldsymbol{\Delta}_{1^{\prime}}(t)\right)\right] \\
& +\beta_{1^{\prime}} q_{1}(t)+N_{1^{\prime}}^{\varepsilon}(t)
\end{aligned}
$$

where we now dropped the bar notation. The complete set of equations can be obtained here and below by cyclic permutation of the indices (i.e. $1 \rightarrow 2,1^{\prime} \rightarrow 2^{\prime}$, etc). The main goal is now to remove the main noise sources, i.e. laser phase noise, $p$, clock noise, $q$, and OB displacement noise, $\boldsymbol{\Delta}$, from the given system of equations.

\subsection{Clock tone transfer chain}

Each OB provides three measurements that can be used to remove noise. However, there is insufficient information to remove the clock noise, $q$. A clock tone transfer chain, as proposed in [7], provides additional information on the SB to cancel clock noise. A sketch of the transfer chain is shown in figure 6.

On the outgoing beam of the distant $\mathrm{S} / \mathrm{C}$, the USO phase noise, $q_{2}$, is amplified by a large integer $\left(m_{2^{\prime}} \sim 10^{2}\right)$ and is imprinted as phase modulation SB via an electro-optic-modulator (EOM). This is then transmitted over $5000000 \mathrm{~km}$ to the receiving S/C. There, the incoming light is interfered with the local laser light that itself is SB-modulated at a slightly different frequency. The resulting SB-SB measurement $s_{1}^{\mathrm{sb}}(t)$ is then given in terms of phase by

$$
\begin{aligned}
s_{1}^{\mathrm{sb}}(t)=\theta_{2^{\prime} 1}[ & \mathcal{D}_{3} p_{2^{\prime}}(t)-p_{1}(t)+h_{1}(t)-\frac{2 \pi}{\lambda_{2^{\prime}}} \mathbf{n}_{3} \cdot \mathcal{D}_{3} \boldsymbol{\Delta}_{2^{\prime}}(t)+\frac{2 \pi}{\lambda_{2^{\prime}}} \mathbf{n}_{3} \cdot \boldsymbol{\Delta}_{1}(t) \\
& \left.+m_{2^{\prime}} \mathcal{D}_{3} q_{2}(t)-m_{1} q_{1}(t)+N_{1}^{\mathrm{opt}, \mathrm{sb}}(t)\right]+\gamma_{1} q_{1}(t)+N_{1}^{\mathrm{s}, \mathrm{sb}}(t),
\end{aligned}
$$




$$
\begin{aligned}
s_{1^{\prime}}^{\mathrm{sb}}(t)=\theta_{31^{\prime}}[ & \mathcal{D}_{2^{\prime}} p_{3}(t)-p_{1^{\prime}}(t)+h_{1^{\prime}}(t)+\frac{2 \pi}{\lambda_{3}} \mathbf{n}_{2} \cdot \mathcal{D}_{2^{\prime}} \boldsymbol{\Delta}_{3}(t)-\frac{2 \pi}{\lambda_{3}} \mathbf{n}_{2} \cdot \boldsymbol{\Delta}_{1^{\prime}}(t) \\
& \left.+m_{3} \mathcal{D}_{2^{\prime}} q_{3}(t)-m_{1^{\prime}} q_{1}(t)+N_{1^{\prime}}^{\mathrm{opt}, \mathrm{sb}}(t)\right]+\gamma_{1^{\prime}} q_{1}(t)+N_{1^{\prime}}^{\mathrm{s}, \mathrm{sb}}(t)
\end{aligned}
$$

where $\gamma_{i}=\frac{\Delta f_{s_{i}}^{\mathrm{sb}}}{f_{\mathrm{ADC}}}$. These signals basically contain the same information as $s_{1}^{\mathrm{c}}(t)$ and $s_{1^{\prime}}^{\mathrm{c}}(t)$, but with the clock noise amplified by the integer factors $m_{i}$. In practice, two SB-SB measurements are performed (upper and lower SB, figure 6) to increase the signal-to-noise ratio. However, both contain redundant information. For the rest of this paper, we will only consider one SB-SB measurement.

\section{Clock noise and laser phase noise removal schemes}

Finally, there are four data streams per OB (namely $s^{\mathrm{c}}(t), s^{\mathrm{sb}}(t), \tau(t)$ and $\left.\varepsilon(t)\right)$. The following procedure can be used to remove laser phase noise and clock noise. The goal is to obtain an intermediate result data stream in the form of

$$
Q(t)=\mathcal{D} p-p+h+\mu-\mu^{\prime},
$$

( $p$ : laser phase noise, $h$ : gravitational wave signal, $\mu$ : 'backlink' fibre noise) where we omit the indices for simplicity. It is therefore necessary to remove all OB motion, clock noise and laser phase noises of the primed lasers. Furthermore, we have to take care that the noise, $\mu$, introduced by the 'backlink'-fibre phase delay only appears as a difference to its counterpart, $\mu^{\prime}$. In the case when the fibre can be assumed to be reciprocal, these terms will cancel [15].

\subsection{OB motion}

First, the OB motion can be removed. Consider the combination

$$
\varepsilon_{1}(t)-\tau_{1}(t)=-2 \theta_{1^{\prime} 1}\left(\frac{2 \pi}{\lambda_{1^{\prime}}} \mathbf{n}_{3} \cdot \boldsymbol{\delta}_{1}(t)-\frac{2 \pi}{\lambda_{1^{\prime}}} \mathbf{n}_{3} \cdot \boldsymbol{\Delta}_{1}(t)\right)+N_{1}^{\varepsilon}(t)-N_{1}^{\tau}(t) .
$$

It can be seen that it is possible to extract the OB motion from laser phase noise and clock noise. Thus,

$$
\begin{aligned}
& \xi_{1}(t) \equiv s_{1}^{\mathrm{c}}(t)-\theta_{2^{\prime} 1} \theta_{1^{\prime} 1} \frac{\lambda_{1^{\prime}}}{\lambda_{2^{\prime}}} \frac{\varepsilon_{1}(t)-\tau_{1}(t)}{2}-\theta_{2^{\prime} 1} \theta_{22^{\prime}} \frac{\lambda_{2}}{\lambda_{2^{\prime}}} \frac{\mathcal{D}_{3} \varepsilon_{2^{\prime}}(t)-\mathcal{D}_{3} \tau_{2^{\prime}}(t)}{2}, \\
& \xi_{1^{\prime}}(t) \equiv s_{1^{\prime}}^{\mathrm{c}}(t)-\theta_{31^{\prime}} \theta_{11^{\prime}} \frac{\lambda_{1}}{\lambda_{3}} \frac{\varepsilon_{1^{\prime}}(t)-\tau_{1^{\prime}}(t)}{2}-\theta_{31^{\prime}} \theta_{3^{\prime} 3} \frac{\lambda_{3^{\prime}}}{\lambda_{3}} \frac{\mathcal{D}_{2^{\prime}} \varepsilon_{3}(t)-\mathcal{D}_{2^{\prime}} \tau_{3}(t)}{2}
\end{aligned}
$$

are free of phase noise due to $\mathrm{OB}$ motion. We further define data streams free of primed laser phase noise:

$$
\begin{aligned}
& \eta_{1}(t) \equiv \theta_{2^{\prime} 1} \xi_{1}(t)-\frac{\theta_{2^{\prime} 2} \mathcal{D}_{3} \tau_{2}(t)-\theta_{22^{\prime}} \mathcal{D}_{3} \tau_{2^{\prime}}(t)}{2} \\
& \eta_{1^{\prime}}(t) \equiv \theta_{31^{\prime}} \xi_{1^{\prime}}(t)-\frac{\theta_{11^{\prime}} \tau_{1^{\prime}}(t)-\theta_{1^{\prime} 1} \tau_{1}(t)}{2}
\end{aligned}
$$




\subsection{Clock noise removal}

It is clear that by using only the measurements described above, it is not possible to remove all clock errors, because the measurements only contain differences between clocks, thus being insensitive to a common-mode error in all clocks. We therefore need to set a reference; in the following, we choose $q_{1}(t):=0$. Thus, the other $q$-terms can be considered as relative clock drifts to the reference. With the use of $\theta_{i j}^{2}=1$ (equation (8)), the $\eta$-data streams take the form

$$
\begin{aligned}
\eta_{1}(t) \sim h_{1}(t)- & p_{1}(t)+\mathcal{D}_{3} p_{2}(t)+\frac{1}{2} \beta_{2} \theta_{22^{\prime}} \mathcal{D}_{3} q_{2}(t)-\frac{1}{2} \beta_{2} \theta_{2^{\prime} 2} \mathcal{D}_{3} q_{2}(t) \\
& +\frac{\mathcal{D}_{3} \mu_{2}(t)-\mathcal{D}_{3} \mu_{2^{\prime}}(t)}{2}, \\
\eta_{1^{\prime}}(t) \sim h_{1^{\prime}}(t)- & p_{1}(t)+\mathcal{D}_{2^{\prime}} p_{3}(t)+\frac{\mu_{1^{\prime}}(t)-\mu_{1}(t)}{2}, \\
\eta_{2}(t) \sim h_{2}(t)- & p_{2}(t)+\mathcal{D}_{1} p_{3}(t)+\alpha_{2} \theta_{3^{\prime} 2} q_{2}(t)+\frac{1}{2} \beta_{3} \theta_{33^{\prime}} \mathcal{D}_{1} q_{3}(t) \\
& -\frac{1}{2} \beta_{3} \theta_{3^{\prime} 3} \mathcal{D}_{1} q_{3}(t)+\frac{\mathcal{D}_{1} \mu_{3}(t)-\mathcal{D}_{1} \mu_{3^{\prime}}(t)}{2}, \\
\eta_{2^{\prime}}(t) \sim h_{2^{\prime}}(t) & -p_{2}(t)+\mathcal{D}_{3^{\prime}} p_{1}(t)+\alpha_{2^{\prime}} \theta_{12^{\prime}} q_{2}(t)-\frac{1}{2} \beta_{2} \theta_{22^{\prime}} q_{2}(t) \\
& +\frac{1}{2} \beta_{2} \theta_{2^{\prime}} q_{2}(t)+\frac{\mu_{2^{\prime}}(t)-\mu_{2}(t)}{2}, \\
\eta_{3}(t) \sim h_{3}(t)- & p_{3}(t)+\mathcal{D}_{2} p_{1}(t)+\alpha_{3} \theta_{1^{\prime} 3} q_{3}(t)+\frac{\mathcal{D}_{2} \mu_{1}(t)-\mathcal{D}_{2} \mu_{1^{\prime}}(t)}{2}, \\
\eta_{3^{\prime}}(t) \sim h_{3^{\prime}}(t) & -p_{3}(t)+\mathcal{D}_{1^{\prime}} p_{2}(t)+\alpha_{3^{\prime}} \theta_{23^{\prime}} q_{3}(t)-\frac{1}{2} \beta_{3} \theta_{33^{\prime}} q_{3}(t) \\
& +\frac{1}{2} \beta_{3} \theta_{3^{\prime} 3} q_{3}(t)+\frac{\mu_{3^{\prime}}(t)-\mu_{3}(t)}{2} .
\end{aligned}
$$

We now make use of the SB information, e.g.

$$
s_{3}^{\mathrm{c}}(t)-s_{3}^{\mathrm{sb}}(t) \sim m_{3} \theta_{1^{\prime} 3} q_{3}(t)+\alpha_{3} q_{3}(t)-\gamma_{3} q_{3}(t),
$$

where the symbol $\sim$ in this context emphasizes that we regard only the main noise sources (lasers, clocks and OB displacement) and neglect, e.g., shot noise, electronic noise, etc. Hence, it becomes possible to remove clock noise. The clock noise free data, $Q(t)$, are then given by

$Q_{1}(t) \equiv \eta_{1}(t)+\beta_{2} \theta_{2^{\prime} 2} \frac{\mathcal{D}_{3} s_{2^{\prime}}^{\mathrm{c}}(t)-\mathcal{D}_{3} s_{2^{\prime}}^{\mathrm{sb}}(t)}{\alpha_{2^{\prime}}+\theta_{12^{\prime}} m_{2^{\prime}}-\gamma_{2^{\prime}}}$,

$Q_{2}(t) \equiv \eta_{2}(t)-\alpha_{2} \theta_{3^{\prime} 2} \frac{s_{2^{\prime}}^{\mathrm{c}}(t)-s_{2^{\prime}}^{\mathrm{sb}}(t)}{\alpha_{2^{\prime}}+\theta_{12^{\prime}} m_{2^{\prime}}-\gamma_{2^{\prime}}}+\beta_{3} \theta_{3^{\prime} 3} \frac{\mathcal{D}_{1} s_{3}^{\mathrm{c}}(t)-\mathcal{D}_{1} s_{3}^{\mathrm{sb}}(t)}{\alpha_{3}+\theta_{1^{\prime} 3} m_{3}-\gamma_{3}}$,

$Q_{3}(t) \equiv \eta_{3}(t)-\alpha_{3} \theta_{1^{\prime} 3} \frac{s_{3}^{\mathrm{c}}(t)-s_{3}^{\mathrm{sb}}(t)}{\alpha_{3}+\theta_{1^{\prime} 3} m_{3}-\gamma_{3}}$

and furthermore

$Q_{1^{\prime}}(t) \equiv \eta_{1^{\prime}}(t)$

$Q_{2^{\prime}}(t) \equiv \eta_{2^{\prime}}(t)-\alpha_{2^{\prime}} \theta_{12^{\prime}} \frac{s_{2^{\prime}}^{\mathrm{c}}(t)-s_{2^{\prime}}^{\mathrm{sb}}(t)}{\alpha_{2^{\prime}}+\theta_{12^{\prime}} m_{2^{\prime}}-\gamma_{2^{\prime}}}+\beta_{2} \theta_{22^{\prime}} \frac{s_{2^{\prime}}^{\mathrm{c}}(t)-s_{2^{\prime}}^{\mathrm{sb}}(t)}{\alpha_{2^{\prime}}+\theta_{12^{\prime}} m_{2^{\prime}}-\gamma_{2^{\prime}}}$,

$Q_{3^{\prime}}(t) \equiv \eta_{3^{\prime}}(t)-\alpha_{3^{\prime}} \theta_{23^{\prime}} \frac{s_{3}^{\mathrm{c}}(t)-s_{3}^{\mathrm{sb}}(t)}{\alpha_{3}+\theta_{1^{\prime} 3} m_{3}-\gamma_{3}}+\beta_{3} \theta_{33^{\prime}} \frac{s_{3}^{\mathrm{c}}(t)-s_{3}^{\mathrm{sb}}(t)}{\alpha_{3}+\theta_{1^{\prime} 3} m_{3}-\gamma_{3}}$. 
All synthesized data combinations $Q_{i}(t)$ yield the analytical form of equation (11), as demanded for TDI input. Thus, the shown algorithm yields clock noise free, and OB motion free, data combinations and, furthermore, keep track of the beatnote sign at each photodetector.

\subsection{Laser phase noise cancellation}

Finally, the above $Q$-combinations can be used as input to the TDI algorithm itself. Application of TDI will remove the laser phase noise by orders of magnitude to a level below the gravitational wave signal. Although we introduced the beatnote signs, the presented algorithm takes care of the laser frequency order and the well-known TDI equations remain invariant:

$$
\begin{aligned}
X(t)=\left[Q_{1^{\prime}}(t)\right. & \left.+\mathcal{D}_{2^{\prime}} Q_{3}(t)+\mathcal{D}_{2^{\prime}} \mathcal{D}_{2} Q_{1}(t)+\mathcal{D}_{2^{\prime}} \mathcal{D}_{2} \mathcal{D}_{3} Q_{2^{\prime}}(t)\right]-\left[Q_{1}(t)+\mathcal{D}_{3} Q_{2^{\prime}}(t)\right. \\
& \left.+\mathcal{D}_{3} \mathcal{D}_{3^{\prime}} Q_{1^{\prime}}(t)+\mathcal{D}_{3} \mathcal{D}_{3^{\prime}} \mathcal{D}_{2^{\prime}} Q_{3}(t)\right] \\
\sim h_{1^{\prime}}(t)+ & \mathcal{D}_{2^{\prime}} h_{3}(t)+\mathcal{D}_{2^{\prime}} \mathcal{D}_{2} h_{1}(t)+\mathcal{D}_{2^{\prime}} \mathcal{D}_{2} \mathcal{D}_{3} h_{2^{\prime}}(t) \\
& -h_{1}(t)-\mathcal{D}_{3} h_{2^{\prime}}(t)-\mathcal{D}_{3} \mathcal{D}_{3^{\prime}} h_{1^{\prime}}(t)-\mathcal{D}_{3} \mathcal{D}_{3^{\prime}} \mathcal{D}_{2^{\prime}} h_{3}(t)+\frac{\mu_{1^{\prime}}(t)-\mu_{1}(t)}{2} \\
& +\frac{\mathcal{D}_{2} \mathcal{D}_{2^{\prime}} \mu_{1}(t)-\mathcal{D}_{2} \mathcal{D}_{2^{\prime}} \mu_{1^{\prime}}(t)}{2}+\frac{\mathcal{D}_{3} \mathcal{D}_{3^{\prime}} \mu_{1}(t)-\mathcal{D}_{3} \mathcal{D}_{3^{\prime}} \mu_{1^{\prime}}(t)}{2} \\
& +\frac{\mathcal{D}_{2} \mathcal{D}_{2^{\prime}} \mathcal{D}_{3} \mathcal{D}_{3^{\prime}} \mu_{1^{\prime}}(t)-\mathcal{D}_{2} \mathcal{D}_{2^{\prime}} \mathcal{D}_{3} \mathcal{D}_{3^{\prime}} \mu_{1}(t)}{2}
\end{aligned}
$$

and other combinations similarly. As can be seen from equation (15), all dominant noise sources are removed while the gravitational wave signal $h$ is preserved. Furthermore, the phase noise $\mu$ entering via light travelling through the 'backlink' fibres appears in each term of equation (15) as a difference with its counterpart $\mu^{\prime}$, as required above.

\section{Discussion and outlook}

We introduced a consistent notation and formulated the LISA-like data streams for the split interferometry configuration. Each OB provides four main data streams containing the gravitational wave signal, TM motion, dominant clock and laser phase noise and further noises, each appearing with different time stamps. This in combination with a clock tone transfer chain for additional data to remove clock noise provides sufficient information to remove the dominant noise sources.

We showed a compliant algorithm to remove $\mathrm{OB}$ motion and clock noise and ensure correct functioning of TDI. Furthermore, we tracked the laser frequency order and showed that the algorithm is designed to be insensitive to the frequency order. The next step is to extend the discussion to second generation TDI and to set up a frequency plan for the whole LISA mission lifetime. Furthermore, a numerical simulation by the modified 'LISA-Code' [16] (used for the Mock LISA data challenge [17]) is in development to confirm the analytical results of this paper.

\section{Acknowledgments}

We gratefully acknowledge support from Deutsches Zentrum für Luft- und Raumfahrt (DLR) with funding of the Bundesministerium für Wirtschaft und Technologie with a decision of the Deutschen Bundestag (DLR project reference no 50 OQ 0601) and thank the Deutsche Forschungsgemeinschaft (DFG) for funding the Cluster of Excellence QUEST-Centre for Quantum Engineering and Spacetime Research. 


\section{References}

[1] The LISA Study Team 1998 Laser Interferometer Space Antenna for the detection and observation of gravitational waves: Pre-phase A report (Max-Planck-Institute for Quantum Optics)

[2] LISA International Science Team 2011 LISA assessment study report (Yellow Book) (European Space Agency) ESA/SRE(2011)3

[3] Thorpe J I, Maghami P and Livas J 2011 Time domain simulations of arm locking in LISA Phys. Rev. D 83122002

[4] Tinto M and Armstrong J W 1999 Cancellation of laser noise in an unequal-arm interferometer detector of gravitational radiation Phys. Rev. D 59102003

[5] Delgado J J E, Garcia-Marin A F, Bykov I, Heinzel G and Danzmann K 2009 Free-space laser ranging and data communication Proc. 6th Workshop on Positioning, Navigation and Communication pp 275-81

[6] Esteban J J, Garcia-Marin A F, Barke S, Peinado A M, Cervantes F G, Bykov I, Heinzel G and Danzmann K 2011 Experimental demonstration of weak-light laser ranging and data communication for LISA Opt. Express 19 15937-46

[7] Barke S, Troebs M, Sheard B, Heinzel G and Danzmann K 2010 EOM sideband phase characteristics for the spaceborne gravitational wave detector LISA Appl. Phys. B 98 33-9

[8] Tinto M, Estabrook F B and Armstrong J W 2004 Time delay interferometry with moving spacecraft arrays Phys. Rev. D 69082001

[9] Shaddock D A, Ware B, Spero R E and Vallisneri M 2004 Postprocessed time-delay interferometry for LISA Phys. Rev. D 70081101

[10] Dhurandhar S V, Nayak K R and Vinet J Y 2002 Algebraic approach to time-delay data analysis for LISA Phys. Rev. D 65102002

[11] Tinto M and Dhurandhar S V 2005 Time-delay interferometry Living Rev. Rel. 84 (http://www.livingreviews. org/lrr-2005-4)

[12] Hellings R W 2001 Elimination of clock jitter noise in spaceborne laser interferometers Phys. Rev. D 64022002

[13] Thorpe J I 2010 LISA long-arm interferometry Class. Quantum Grav. 27084008

[14] Heinzel G, Esteban J J, Barke S, Otto M, Wang Y and Danzmann K 2011 Auxiliary functions of the LISA laser link: ranging, clock noise transfer and data communication Class. Quantum Grav. 28094008

[15] Fleddermann R, Steier F, Troebs M, Bogenstahl J, Killow C, Heinzel G and Danzmann K 2009 Measurement of the non-reciprocal phase noise of a polarization maintaining single-mode optical fiber J. Phys.: Conf. Ser. 154012022

[16] Petiteau A, Auger G, Halloin H, Jeannin O, Plagnol E, Pireaux S, Regimbau T and Vinet J Y 2008 LISACode: a scientific simulator of LISA Phys. Rev. D 77023002

[17] Babak S et al 2008 Report on the second Mock LISA data challenge Class. Quantum Grav. 25114037 